\title{
A PRESENÇA E AS POTENCIALIDADES DA AGRICULTURA FAMILIAR NA AMÉRICA LATINA E NO CARIBE
}

\section{PRESENCE AND POTENTIALS OF FAMILY FARMING IN LATIN AMERICA AND THE CARIBBEAN}

\author{
Sergio Schneider \\ Universidade Federal do Rio Grande do Sul - Porto Alegre - RS - Brasil
}

\begin{abstract}
Resumo: A agricultura familiar constitui o grupo social majoritário de unidades produtivas do meio rural latino-americano. Nos anos recentes, houve avanços importantes em relação ao reconhecimento de seu papel no desenvolvimento rural e na superação da pobreza. Várias organizações internacionais lançaram estudos para afirmar o papel estratégico dos pequenos produtores na segurança alimentar. Neste artigo, apresenta-se a agricultura familiar na América Latina e no Caribe e se discute a sua presença em diferentes países mediante o uso de dados estatísticos disponíveis, assim como se analisa as suas potencialidades para fortalecer o desenvolvimento rural. O trabalho inicia com uma discussão sobre os elementos que contribuíram para o interesse crescente dos estudiosos e formuladores de políticas na agricultura familiar e, na sequência, analisa-se suas características e a distribuição em diferentes países. 0 trabalho conclui que a agricultura familiar possui um papel central para o desenvolvimento rural da região latinoamericana e encerra com uma análise sobre as principais potencialidades em face do quadro político e institucional.

Palavras-chave - Agricultura familiar. America Latina. Desenvolvimento rural. Políticas públicas.
\end{abstract}

Abstract: Family farming represent the major social group of farm units in rural Latin American. In recent years, there have been important advances in relation to the recognition of its role in rural development and to overcome poverty. Several international organizations have launched studies to support the strategic role of smallholder farmers in food security. This article presents the family farming in Latin America and the Caribbean and discusses its presence in different countries through the use of available statistical data, as well as analyzing their potential to enhance rural development. The paper begins with a discussion of the main features that contributed to the growing interest of scholars and policy makers in family farming and further one analyse its characteristics and distribution along different countries. The study concludes that family farming has a central role in rural development in Latin American 
and end up with an analysis of the main strengths in the current political and institutional framework.

Key words - Family farming. Latin America. Rural development. Public policies

\section{Introdução}

Como a agricultura vai produzir alimentos mais saudáveis e de forma ambientalmente sustentável para abastecer uma população cada vez mais urbanizada? Quem vai produzir esses alimentos, quais agricultores e sistemas de produção são os mais recomendados para dar conta desse desafio? Essas são perguntas para as quais ainda não se tem resposta, mas já se sabe que esses são alguns dos grandes temas do presente milênio.

Mas, a julgar pelos estudos e pesquisas que têm sido publicados nos últimos anos (PRETTY et.al., 2010; HLPE, 2012; IAASTD, 2009), é possível antever uma certeza: os pequenos agricultores do mundo, aqueles que vivem e trabalham em lotes de terra de tamanho reduzido e manejam quantidades restritas de recursos produtivos (águas, florestas, pastagens e outros ecossistemas), mediante o uso predominante do trabalho familiar, indubitavelmente serão parte da solução e da resposta para as questões acima (BELIERES et.al, 2013; CIRAD, 2013).

Há um consenso de que os pequenos produtores, também chamados de agricultores familiares, terão um papel central a cumprir no alcance dos Objetivos do Desenvolvimento Sustentável, lançados em 2015 pela Organização das Nações Unidas. Estudos de diferentes matizes teóricas e autores com distintas orientações políticas, como Wiggins (2009), Pretty et alii (2011); Larson et alii (2012) e Schutter (2014), destacam que a pequena agricultura poderá ser estratégica no contexto da pós-revolução verde, em que se busca uma "intensificação sustentável" (sustainable intensification) que seja capaz de manter e de aumentar a produtividade do trabalho de tal forma a gerar excedentes alimentares sem comprometer a sustentabilidade.

Não se trata de nenhum mito ou falácia, como erroneamente tem sido propalado no Brasil por aqueles que defendem o modelo convencional de agricultura, baseado na grande propriedade territorial, no trabalho assalariado, nas monoculturas e em todo o pacote de venenos e a parafernália agroquímica subjacente. Basta consultar as evidências científicas mais atualizadas disponíveis, tais como os 
trabalhos recentes de Reganold e Watcher (2016), assim como Ponisio et.al. (2016), que reforçam as conclusões anteriores do impactante estudo de Godfray et.al. (2010) de que é possível abastecer e alimentar a população atual e futura do planeta (os 9,5 bilhões de bocas a alimentar em 2050) com base em sistemas de produção baseados no trabalho familiar e sem o uso dos agroquímicos. Claro que isso não significa excluir ou demonizar a agricultura não familiar, que certamente continuará a ter um papel igualmente relevante.

Mas a questão central a reter desses estudos é que a agricultura familiar não é residual ou sem importância do ponto de vista da produção agroalimentar. Várias organizações internacionais lançaram estudos nos últimos anos para afirmar o papel estratégico da pequena agricultura no desenvolvimento rural e na segurança alimentar. A Organização das Nações Unidas para Alimentação e Agricultura (GLADEK et ali, 2016; Lowder, at alii, 2014; 2016) publicou que do total de 570 milhões de unidades produtivas que existem no mundo, nada menos do que 500 milhões são de pequenos agricultores, também chamados de agricultores familiares'. A FAO (2014a; 2015) estima que "a agricultura familiar é de longe a forma mais prevalente de agricultura no mundo. Estimativas sugerem que ela ocupam cerca de $70-80 \%$ das terras agrícolas e produzem mais de $80 \%$ dos alimentos do mundo em termos de valor" (2014a, p.11). O estudo do United Nations Procurement Division/Programa das Nações Unidas para o Desenvolvimento (2011) sugere que a agricultura familiar poderá ser decisiva para gerar resiliência ambiental e fortalecer os modos de vida rurais de tal forma que sejam mais sustentáveis e aptos a enfrentar as transformações ambientais geradas pelas mudanças climáticas. $O$ relatório do International Fund for Agricultural Development/United Nations Enviroment Programme (2013) destacou o papel estratégico dos pequenos produtores para a segurança alimentar e o meio ambiente.

\footnotetext{
1 Nagayets (2005) estima que existam cerca de 525 milhões de explorações agrícolas de todos os tamanhos no mundo, usando dados do censo agrícola da FAO. Hazell, Poulton, Wiggins, e Dorward (2010) utilizaram dados semelhantes para afirmar que há cerca de 500 milhões de pequenas propriedades com menos de 2 hectares. $O$ trabalho recente de Graeub et. alii. (2015) amplia estas proporções e mostra que 98\% dos estabelecimentos agropecuários do mundo são de agricultores familiares, que detém $53 \%$ da terra e produzem pelo menos $53 \%$ dos alimentos no mundo.
} 
Não restam dúvidas, portanto, quanto à importância multidimensional dos pequenos produtores para enfrentar alguns dos desafios mais urgentes da humanidade, que estão plasmados nos Objetivos do Desenvolvimento Sustentável, estabelecidos em 2015 pela ONU. Essa constatação possui vários desdobramentos, sendo um dos mais evidentes $o$ fato de que as organizações internacionais multilaterais do sistema Nações Unidas reconhecem o papel e o potencial da agricultura familiar no processo de desenvolvimento, razão pela qual, em 2014, celebrou-se o Ano Internacional da Agricultura Familiar.

Portanto, parece chegado o momento em que já não se deve tratar mais os pequenos produtores (proprietários) como sinônimo de pobreza ou de atraso, ou fadados ao desaparecimento inexorável. Por isso, é importante mudar a própria terminologia que vem sendo utilizada, deixando de referi-los como pequenos produtores para passar a chama-los de agricultores familiares ou unidades produtivas familiares (SCHNEIDER, 2014; RAMOS, et. al. 2014). Não se trata apenas de mudar o nome, uma vez que há implicações de natureza conceitual e teórica, mas, acima de tudo, indicar que essa categoria social pode ter um papel ativo e estratégico nos processos de mudança social rural.

$\mathrm{Na}$ América Latina e no Caribe-ALC, a agricultura familiar tem ampliado o reconhecimento sobre as potencialidades dessa categoria social para o desenvolvimento econômico de vários países da região (CEPAL/FAO/IICA, 2013; ECHENIQUE y ROMERO, 2009; BARRIL y ALMADA; 2007). A agricultura familiar gera empregos e renda no meio rural, mas também responde por uma parcela significativa do abastecimento alimentar, especialmente em nível local e regional2. $\mathrm{Na}$ maioria dos países da América Latina e do Caribe, a agricultura familiar dá uma contribuição expressiva à produção agroalimentar, tanto para o mercado interno como para as exportações de commodities e de outros produtos.

\footnotetext{
2 Estudo recente de D'Odorico et al. (2014) mostra que a quantidade de alimentos comercializados no mercado internacional mais do que duplicou entre 1986 e 2009, registrando um aumentou de 15\% em 1986 para 23\% em 2009. Mas o mais interessante do estudo de D'Odorico et al. (2014, p.461) é o fato de que $77 \%$ dos alimentos produzidos no mundo ainda são consumidos em mercados nacionais e, sobretudo, locais. Apenas cerca de $23 \%$ dos alimentos produzidos para consumo humano são comercializados internacionalmente.
} 
Neste artigo, pretende-se apresentar a agricultura familiar na América Latina e no Caribe e discutir a sua presença em diferentes países, assim como suas potencialidades para o desenvolvimento rural nas regiões. Na segunda seção, apresenta-se os principais elementos que contribuíram para o interesse crescente dos estudiosos e formuladores de políticas sobre a agricultura familiar. Na terceira seção, apresenta-se as características e a distribuição da agricultura familiar em diferentes regiões da ALC e nos respectivos países. Na seção final, discute-se as principais potencialidades da agricultura familiar para o desenvolvimento a partir de ideias e de propostas recolhidas em documentos internacionais e que foram alvo de discussão nos anos recentes, particularmente em 2014, que foi o Ano Internacional da Agricultura Familiar. 


\section{A presença da agricultura familiar na América Latina e no Caribe}

Nas últimas duas décadas, a América Latina e o Caribe passaram por mudanças significativas em termos econômicos, sociais e políticos, tornando a região significativamente distinta do que era na década de 1980. Estudiosos, instituições internacionais e policy makers concordam que a região latino-americana e caribenha passou a sofrer mais intensivamente os efeitos da globalização econômica internacional, cujas implicações vão desde a maior abertura comercial das economias nacionais, o aumento de fluxos financeiros e a revalorização dos recursos primários como a produção agroalimentar e a mineração (PIADAL, 2013). Mas essas mudanças também possuem impactos sociais e ambientais, que são expressos nos fortes processos migratórios inter e intraregionais, assim como sobre o meio ambiente e os recursos naturais de forma geral.

No período recente, a América Latina e o Caribe estão passando por um processo de discussão e análise sobre o papel e o lugar da agricultura familiar no desenvolvimento social e econômico dos países (BAUMEISTER, 2012). Trata-se de um processo novo, que se iniciou na metade da década de 1990 no Brasil, foi ganhando espaço e se irradiou a partir dos anos 2000 para outros países da região. Iniciativas regionais como a criação da Rede Especializada da Agricultura FamiliarREAF entre os países que integram o Mercado Comum do Sul, em 2004, e mais recentemente iniciativas na região Centro Americana, com destaque para o Plan de Agricultura Familiar-PAF de El Salvador têm sido importantes para difundir o conceito e a compreensão de seu significado (REAF, 2011).

Segundo Leporati et alii. (2014, p.35), a agricultura familiar soma cerca de $81 \%$ dos estabelecimentos agrícolas da ALC, responde entre $27 \%$ e $67 \%$ do total da produção alimentar por país, ocupa entre $12 \%$ e $67 \%$ da superfície agropecuária e gera entre $57 \%$ e $77 \%$ do emprego agrícola na região (FAO/BID, 2007; FAO, 2012). Estes dados indicam, ainda sem a precisão estatística desejada, que a agricultura familiar tem papel inquestionável na produção primária, na segurança alimentar e no desenvolvimento econômico em geral da ALC.

Mas a contribuição da agricultura familiar não é apenas econômica. No que concerne aos aspectos sociais e demográficos, a agricultura familiar contribuiu de modo decisivo para a manutenção das 
famílias no meio rural. As comunidades rurais em que se verifica a presença da agricultura familiar possuem vida social ativa, que, muitas vezes, reflete-se em dinâmicas locais virtuosas. A agricultura familiar também é importante para as mulheres e os jovens, pois o acesso à terra e aos ativos produtivos são recursos fundamentais para garantia de seus meios de vida nos casos em que os homens migram para trabalhar fora da agricultura (PAULILO, 2013).

Entre os fatores que têm contribuído com o reconhecimento sobre a importância da agricultura familiar, pode-se destacar os logros com a redução da pobreza e a melhoria dos indicadores econômicos e sociais a partir das políticas de favorecimento aos agricultores familiares (SILVA, GÓMEZ; CASTAÑEDA, 2009). Vale notar que a América Latina é uma das regiões no mundo que tem se destacado na redução da fome e da pobreza. A combinação de crescimento econômico, de estabilidade política e institucional e de incentivos para a agricultura e para o desenvolvimento rural foram apontados pelo recente relatório da FAO, Fundo Internacional de Desenvolvimento Agrícola e Programa Mundial de Alimentos (2013) sobre o estado da segurança alimentar como fatores que contribuíram para esse alcance.

Há outros elementos que podem e devem ser mencionados, entre eles, o fato de que o final das ditaduras na América Latina representou a retomada das ações e da capacidade de mobilização das organizações e dos atores sociais da sociedade civil, o que contribuiu para a retomada da organização dos agricultores familiares em movimentos, sindicatos, associações, cooperativas e outras formas (BERDEGUÉ, et alii. 2012). Essas organizações passaram a ter um papel importante na mobilização social e na apresentação de agendas políticas a favor da agricultura familiar na região. Um terceiro fator que joga um papel importante na expansão do reconhecimento e da legitimidade da agricultura familiar na ALC são as políticas públicas. Apesar de haver uma grande diversidade de políticas que beneficiam os agricultores familiares, que varia segundo os distintos países, a ação do Estado tem sido decisiva em favor da agricultura familiar.

Não obstante esses avanços, é preciso reconhecer que há lacunas e limitações que precisam ser superadas. Ainda resta um esforço a ser feito para melhorar a definição e a compreensão teórica e política da agricultura familiar, no sentido de esclarecer as implicações de usar tipologias ou trabalhar com definições genéricas para a elaboração de 
políticas públicas. Outro fator limitante refere-se à disponibilidade de dados e informações sobre a agricultura familiar, pois as atualizações censitárias são precárias em muitos países e nem sempre há disponibilidade de dados específicos sobre a agricultura familiar (SCHNEIDER, 2013; 2014).

\section{Controvérsias conceituais e a busca de uma definição operacional}

Poucas controvérsias têm sido tão duradouras e difíceis de serem superadas quanto a definição sobre o estatuto teórico dos produtores rurais que atualmente se denominam agricultores familiares.

Entre as inconformidades que fazem permanecer a polêmica, está a própria utilização de terminologias (RAMOS, et.al. 2014). Quando se menciona, se ouve ou se lê termos como pequenos produtores agrícolas/rurais ou camponeses ou agricultores familiares logo se imagina um empreendimento econômico que é conduzido por uma família, que tanto trabalha e executa a maior parte das atividades e tarefas, como também se apropria dos resultados dessa operação.

Nada mais equivocada, teórica e politicamente imprecisa do que imaginar que pequena produção, o campesinato e a agricultura familiar sejam a mesma coisa. Em geral, esses três termos se referem a uma mesma categoria social, que pode ser encontrada em áreas rurais dos mais diversos países e regiões subnacionais da ALC (BENGOA, 2002). Mas em cada país e/ou região há denominações locais para identificar as pessoas e famílias que podem ser pequenos produtores agrícolas/rurais, camponeses ou agricultores familiares. Portanto, aqui reside uma primeira distinção entre estes três termos: eles se referem a uma categoria social que varia segundo países e regiões e podem ainda receber denominações regionais específicas 3 .

Os agricultores familiares constituem uma categoria social economicamente diversa e socialmente heterogênea. Trata-se de uma forma particular de organização de trabalho e de produção que existe e se reproduz a partir de distintas estratégias produtivas agrícolas e não

\footnotetext{
3 No Sul do Brasil, por exemplo, os agricultores familiares são denominados como colonos. No nordeste eles são chamados de sitiantes (SCHNEIDER E NIEDERLE, 2010). Nas regiões andinas, em que todos os pequenos produtores são chamados de campesinos, a distinção local é dada segundo a etnia indígena de origem. No México, por exemplo, muitos recebem a denominação de hegidos.
} 
agrícolas em consonância com o contexto social e econômico em que estão inseridos. Sua reprodução ascendente ou descendente não decorre apenas de um ato voluntário como uma política pública ou mesmo um desejo individual ou familiar. Como formas sociais de trabalho e de produção, sua reprodução social está condicionada por fatores internos que estão relacionados ao modo concreto como as famílias gestionam seus recursos produtivos (terra, capital, tecnologia, etc), tomam decisões de investimento e dispêndio, alocam o trabalho de seus membros e aderem aos valores culturais do grupo a que pertencem. Seu sucesso e sua capacidade de reprodução social vão depender da forma como os recursos produtivos, especialmente terra, água e sementes forem geridos no interior da unidade de produção e das decisões e opções que foram tomadas pelos membros do grupo doméstico. Essa forma de gestão e os mecanismos de tomada de decisão ocorrem em meio a contextos que podem restringir, facilitar ou mesmo neutralizar o futuro dessas unidades. Portanto, um dos desafios aos agricultores familiares consiste em desenvolver mecanismos e dispositivos para reduzir os riscos e as incertezas associadas.

Mas os agricultores familiares também não conseguem escapar ao contexto social e econômico em que vivem e pelo qual estão condicionados, às vezes, até subordinados. Entre estes condicionantes estão as demandas urbanas cada vez mais fortes por alimentos saudáveis, mas também pela preservação das paisagens, do solo, da água e da biodiversidade. As inovações tecnológicas também são condicionantes, que podem tanto reduzir o papel da terra quanto da mão de obra nos processos produtivos e, assim, serem decisivas para maior competividade das unidades produtivas. Em resumo, a reprodução da agricultura familiar decorre de processos sociais e econômicos mais gerais e depende de múltiplos fatores, endógenos e externos que, em resumo, configuram uma construção social e política 4 .

Qual seria, então, a distinção fundamental entre agricultura familiar e os outros dois termos, pequena produção e campesinato? Trata-se de controvérsia que já "consumiu rios de tinta", mas é possível afirmar que a diferença entre agricultura familiar e pequena produção reside no fato de que a primeira se refere a uma atividade produtiva

\footnotetext{
4 Para uma discussão teoricamente informada sobre a questão camponesa e da agricultura familiar, consultar, entre outros, Friedmann (1978); Ellis (1988) e Ploeg (2008; 2013).
} 
(agricultura) que é executada por um grupo social (família) ligado por laços de consanguinidade e parentesco e a segunda se refere à escala que possui um determinado produtor, neste caso agrícola (embora, pequeno produtor não necessariamente seja uma produtor agrícola ou que vive no rural). É muito frequente que a escala de pequeno porte esteja ligada ao tamanho da área de terra disponível (própria ou não, ou ainda apenas aquela utilizada para produzir, a área útil), mas também pode se referir à intensidade de uso de outros fatores, especialmente tecnologia e capital. Portanto, pequeno produtor é todo aquele que produz em uma escala econômica reduzida, não importando se é para autoaprovisionamento (autoconsumo) ou para a venda (comercial).

O uso do critério de tamanho de área de terra tornou-se uma unidade de medida internacionalmente aceita para definir um pequeno produtor, o que permite comparações entre países e, sobretudo, uma enumeração quantitativa (NAGAYETS, 2005; GARNER e O CAMPOS, 2014). Conforme apontam Hazell e Rahman (2014), esse critério tornou-se decisivo para a elaboração de estatísticas internacionais comparativas. Mas, é cada vez mais consensual entre os estudiosos internacionais que os indicadores de escala de produção (produtividade e renda) são pouco úteis para compreender e explicar as possibilidades de desenvolvimento dos pequenos produtores (CONWAY, 2014; HAYAMI, 2002).

Malgrado as vantagens estatísticas em se utilizar a definição pequena produção, este termo tornou-se questionável porque o tamanho da área de terra diz muito pouco sobre as condições de produção e de reprodução dos produtores. Um pequeno produtor com até dois ha de terra pode ser tanto considerado economicamente inviável como adequado, a depender da forma como a terra é utilizada, o tipo de produto cultivado, a tecnologia, o acesso a ingressos não agrícolas, etc. Não raro, foi por causa desses critérios que durante muito tempo se associou pequena produção com pobreza rural e, portanto, inviabilidade econômica.

Em relação ao termo/palavra camponeses ou campesinato, a distinção é um pouco mais complexa, pois os agricultores familiares de hoje não deixam de ter suas raízes camponesas ${ }^{5}$. O campesinato

\footnotetext{
5 Para uma revisão da literatura sobre os aportes teóricos sobre "campesinos y pequeños productores" na América Latina ver Wolf (1955); Schultz, (1964); CEPAL
} 
pressupõe uma comunidade rural e um modo de vida relativamente autônomo em relação às cidades e aos mercados. O sociólogo francês Henri Mendras afirmou que a coletividade (comunidade) local e as relações de solidariedade e reciprocidade são centrais para caracterizar as sociedades camponesas. Já o antropólogo americano Eric Wolf se referia aos camponeses como grupos sociais com pouca diferenciação interna, reduzida mobilidade social e relações parciais com as cidades e os mercados. Por caminhos e visões teóricas distintas, ambos chegaram a conclusões similares sobre os camponeses e suas relações com a sociedade e os mercados: à medida que a pequena comunidade rural ou povoado isolado reduza sua influência (isso não quer dizer que desapareça) sobre a reprodução social e econômica dos camponeses e as suas relações externas (sejam elas tecnológicas, culturais, informacionais, mercantis, etc) passam a ser apropriadas, redefinidas e mesmo resignificadas, os camponeses se transformam e mudam as suas características sociais. Deixam de ser quase isolados e semiautônomos e passam a se inserir na dinâmica social mais ampla.

Para superar essas limitações, o termo agricultura familiar vem sendo crescentemente utilizado por estudiosos, policy makers e agentes internacionais. Todo agricultor familiar é detentor de um pequeno pedaço de terra, mas isto não significa que ele seja ineficiente e nem mesmo pobre. Eficiência e desempenho econômico são critérios relativos que estão associados a um determinado modo de utilização dos fatores de produção. A pobreza, especialmente rural, ainda que muito presente em situações nas quais se encontra pequenos produtores rurais não se explica apenas pelo fato de serem pequenos no que se refere ao tamanho da terra disponível. Os fatores que determinam a pobreza rural também são multivariados, mas não se explicam apenas pelo tamanho de área de terra disponível (DE JANVRY Y SADOULET, 2001; LIPTON; 2006).

A utilização do termo agricultura familiar ao invés de pequeno produtor permite ampliar a definição, pois supera o critério quantitativo de área de terra, e agrega a variável trabalho. Assim, o agricultor familiar é todo produtor agrícola/rural que utiliza predominantemente o trabalho da sua família para a execução das atividades que compreendem seu empreendimento. Essa definição acaba por ampliar o

(1984); Warman (1988); Bengoa (2003); Schejtman (2008); Schneider and Escher (2012). 
escopo de quem integra essa categoria, retirando o viés da escala (pequena) e a associação à ineficiência e à pobreza. Isso permite dizer que nem sempre um agricultor familiar é um pequeno produtor e, menos ainda, que é pobre.

No quadro a seguir, pode-se observar algumas definições da categoria agricultura familiar em alguns países selecionados da América Latina, segundo as variáveis de limite de área/superfície de terra, tipo de trabalho, gestão da atividade, renda e local de residência.

Cuadro 1. Denominación y criterios de la categoría "agricultor familiar" en algunos países (FAO, 2012)

\begin{tabular}{|c|c|c|c|c|c|c|}
\hline Países & $\begin{array}{c}\text { Denominació } \\
\mathrm{n}\end{array}$ & $\begin{array}{c}\text { Superficie y } \\
\text { capital }\end{array}$ & Trabajo & $\begin{array}{l}\text { Gestión o } \\
\text { actividad }\end{array}$ & Renta & $\begin{array}{c}\text { Residenci } \\
\text { a }\end{array}$ \\
\hline $\begin{array}{l}\text { Argentin } \\
\text { a }\end{array}$ & $\begin{array}{l}\text { Pequeño } \\
\text { Productor } \\
\text { Agropecuario }\end{array}$ & $\begin{array}{l}\text { Límite } \\
\text { superior } \\
\text { por región }\end{array}$ & $\begin{array}{l}\text { Sin } \\
\text { asalariado } \\
\text { permanente } \\
\end{array}$ & $\begin{array}{l}\text { Trabajo } \\
\text { directo en } \\
\text { la finca }\end{array}$ & Nada dice & Nada dice \\
\hline Brasil & $\begin{array}{l}\text { Agricultor } \\
\text { Familiar }\end{array}$ & $\begin{array}{l}\text { Hasta } 4 \\
\text { módulos } \\
\text { fiscales }\end{array}$ & $\begin{array}{l}\text { Hasta } 1 \text { o } 2 \\
\text { salariado(s) } \\
\text { permanente( } \\
\text { s) }\end{array}$ & $\begin{array}{l}\text { Gestión } \\
\text { familiar }\end{array}$ & $\begin{array}{l}80 \% \text { y } \\
\text { luego } 70 \% \\
\text { de renta } \\
\text { familiar } \\
\text { de la finca }\end{array}$ & $\begin{array}{l}\text { Residir en } \\
\text { la finca o } \\
\text { cerca) }\end{array}$ \\
\hline Chile & $\begin{array}{l}\text { Agricultor } \\
\text { familiar } \\
\text { campesino }\end{array}$ & $\begin{array}{l}\text { Hasta } 12 \\
\text { ha de riego } \\
\text { básico y } \\
\text { activos < } \\
96000 \text { USD }\end{array}$ & $\begin{array}{l}\text { Familiares } \\
\text { trabajan } \\
\text { directament } \\
\text { e la tierra }\end{array}$ & $\begin{array}{l}\text { Trabajar } \\
\text { directament } \\
\text { e en la finca }\end{array}$ & $\begin{array}{l}\text { Renta } \\
\text { principal } \\
\text { de la } \\
\text { unidad } \\
\text { agrícola }\end{array}$ & Nada dice \\
\hline Colombia & $\begin{array}{l}\text { Unidad } \\
\text { Agrícola } \\
\text { Familiar- } \\
\text { UAF }\end{array}$ & $\begin{array}{l}\text { UAF con } \\
\text { patrimonio } \\
\text { familiar }\end{array}$ & $\begin{array}{l}\text { Familiar } \\
\text { y con } \\
\text { derecho a } \\
\text { empleados } \\
\text { externos }\end{array}$ & $\begin{array}{l}\text { agropecuari } \\
\text { a, acuícola } \\
\text { y forestal }\end{array}$ & Sin limite & Nada dice \\
\hline Paraguay & $\begin{array}{l}\text { Agricultura } \\
\text { Familiar } \\
\text { Campesina }\end{array}$ & $\begin{array}{l}\text { Sin ningún } \\
\text { limite }\end{array}$ & $\begin{array}{l}\text { Familiar y } \\
\text { hasta con } \\
20 \\
\text { trabajadores } \\
\text { temporales }\end{array}$ & $\begin{array}{l}\text { Gestión por } \\
\text { la familia }\end{array}$ & Sin limite & $\begin{array}{l}\text { En la } \\
\text { propia } \\
\text { unidad } \\
\text { familiar o } \\
\text { en } \\
\text { comunida } \\
\text { des } \\
\text { cercanas }\end{array}$ \\
\hline Uruguay & $\begin{array}{l}\text { Productor } \\
\text { Familiar }\end{array}$ & $\begin{array}{l}\text { Hasta } 500 \\
\text { ha (índice } \\
100 \\
\text { Coneat) }\end{array}$ & $\begin{array}{l}\text { Familiar y } \\
\text { hasta } 2 \\
\text { salarios } \\
\text { permanente } \\
\text { s } \\
\text { o } 500 \\
\text { jornadas/añ } \\
\text { o }\end{array}$ & $\begin{array}{l}\text { Gestión } \\
\text { familiar o } \\
\text { jefe de } \\
\text { familia }\end{array}$ & $\begin{array}{l}\text { Renta } \\
\text { principal } \\
\text { de la finca } \\
\text { o jornada } \\
\text { laboral en } \\
\text { fincas }\end{array}$ & $\begin{array}{l}\text { Hasta } \\
50 \mathrm{~km} \text { de } \\
\text { la finca }\end{array}$ \\
\hline
\end{tabular}


Fuentes: Sabourin, Samper e Sotomayor, 2014, elaboración a partir de FAO, 2012b, CEPAL et al, 2013 ; Salcedo y Guzman, 2014 y de Argentina SAGPyA 1998 y Proinder 2004; Brasil: Ley 11.326, 2006; Chile: Instituto de Desarrollo Agropecuario-INDAP; Colombia: Ley 160 / 1994; Paraguay : Ley N²419, Instituto Nacional de Desarrollo Rural y Tierra, 2004; Uruguay: Ministerio Ganadería Agrícola y Pesca - MGAP, 2008, Registro de Productor Familiar, 2009

Em um trabalho de revisão sobre as distintas definições de agricultura familiar, Garner e O Campos (2014) encontraram 36 distintas definições e entendimentos. Portanto, não há consenso entre os estudiosos e os policy makers sobre o alcance e o significado da agricultura familiar. A FAO adotou uma definição operacional (working definition) de agricultura familiar, elaborada pelo International Steering Committee for the International Year of Family Farming, que inclui todas as atividades agrícolas de base familiar praticadas na silvicultura, pesca, produção agrícola, pastoralista e na aquicultura operada e gestionada por uma família que utiliza predominantemente mão-de-obra familiar, incluindo as das mulheres e dos homens. A família e a unidade produtiva estão unidas, co-evoluem e combinam funções econômicas, ambientais, sociais e culturais (GARNER Y O CAMPOS, 2014, p.21; PERACl, 2011 ).

\section{Algumas características da agricultura familiar na América Latina e no Caribe}

$\mathrm{Na} A L C$, a agricultura familiar vem adquirindo um papel central no desenvolvimento social e econômico da região (CHIRIBOGA, 2006; SOTO BAQUERO et. al.; 2007). Por suas características, a agricultura familiar é o principal reservoir de mão de obra e força de trabalho do setor agrícola e do espaço rural de muitos países da ALC. O estudo de Leporati et ali (2014) indica que na região ALC o número de estabelecimentos agropecuários familiares seja de 20,4 milhões de estabelecimentos. Nesse número absoluto de estabelecimentos, estima-se que vivem em torno de 60 milhões de pessoas.

A Tabela 1 mostra que em todas as regiões que compõem o território da ALC, a participação da agricultura familiar é extremamente relevante. Segundo o citado estudo, $9 \%$ dos estabelecimentos familiares (1.507.757) encontram-se na região do Caribe, $35 \%$ (5.883.205) estão localizados na América Central e México e o restante de 56\% 
(9.205.875) encontra-se na América do Sul (LEPORTATI et al., 2014). Esse número total de estabelecimentos familiares, por sua vez, corresponde a $81,30 \%$ dos estabelecimentos agropecuários da região, os quais produzem - com variações entre os países - entre $27 \%$ e $67 \%$ do total da produção alimentar nacional, ocupando entre $12 \%$ e $67 \%$ da área/superfície agrícola da região. O percentual de unidades agropecuárias familiares em relação ao total de unidades agropecuárias varia de 78,60\% na América Central e México até 88,40\% no Caribe. 
Tabela 1. Proporção de unidades familiares sobre o total de estabelecimentos agropecuários na América Latina e no Caribe, segundo grandes regiões

\begin{tabular}{cccc}
\hline Grande Região & $\begin{array}{c}\mathbf{N}^{\circ} \text { de Total } \\
\text { Estabelecimentos }\end{array}$ & $\begin{array}{c}\mathbf{N}^{\circ} \text { de Unidades } \\
\text { Familiares }\end{array}$ & $\begin{array}{c}\text { \% Unidades Familiares } \\
\text { sobre o total de Estab. }\end{array}$ \\
\hline $\begin{array}{c}\text { Caribe } \\
\begin{array}{c}\text { América. Central } \\
\text { e México }\end{array}\end{array}$ & 1.704 .651 & 1.507 .757 & $88,40 \%$ \\
Países Andinos & 5.486 .831 & 5.883 .205 & $78,60 \%$ \\
Cone Sul & 6.144 .774 & 4.051 .342 & $79,80 \%$ \\
\hline Total & 20.414 .539 & 16.596 .837 & $83,90 \%$ \\
\hline
\end{tabular}

Fonte: elaboração própria do autor a partir de Leporati et. al (2014).

$\mathrm{Na}$ tabela 2, verifica-se que a área média que os agricultores familiares da região da ALC utilizam para desenvolverem suas atividades é de apenas 13,64 ha. Como pode-se perceber, os países do Cone Sul do continente são os que possuem maiores áreas de terra $(47,02 \mathrm{ha})$, seguidos pelos países da América Central (3,13ha) e Andinos (3,09ha). Vale destacar o acesso limitado a este ativo por parte dos agricultores familiares da região do Caribe, os quais possuem, em média, apenas 1,33ha.

Tabela 2. Superfície e tamanho médio dos estabelecimentos do setor agropecuário e na agricultura familiar na América Latina e Caribe, por grandes regiões

\begin{tabular}{ccccc}
\hline \multirow{2}{*}{$\begin{array}{c}\text { Grandes } \\
\text { Regiões }\end{array}$} & Superfície & Média por Estab. & Superfície & $\begin{array}{c}\text { Média por } \\
\text { Estab. }\end{array}$ \\
\cline { 2 - 5 } Caribe & 403.435 & 2,59 & 93.578 & 1,33 \\
$\begin{array}{c}\text { América Central } \\
\text { México }\end{array}$ & 8.014 .679 & 13,85 & 1.137 .100 & 3,13 \\
$\begin{array}{c}\text { Países Andinos } \\
\text { Cone Sul }\end{array}$ & 101.803 .749 & 19,08 & 11.855 .572 & 3,09 \\
\hline Total & 558.759 .298 & 195,07 & 143.618 .362 & 47,02 \\
\hline
\end{tabular}

Fonte: elaboração própria do autor a partir de Leporati et. al (2014). 
De uma maneira geral, na maioria dos casos latino-americanos em que os dados estatísticos que quantificam a agricultura familiar advém dos Censos, verifica-se apenas uma estratificação da superfície física do universo dos estabelecimentos agropecuários. Ainda são raros os países em que há estudos que permitam trabalhar com outras variáveis, tais como valor bruto da produção (FAO/CEPAL/IICA, 2013). Contudo, apesar dessas limitações dos dados, estima-se que a agricultura familiar representa uma proporção de $75 \%$ do total das unidades produtivas em todos os países da região, podendo ultrapassar a proporção de $90 \%$ em alguns destes (FAO/CEPAL/IICA, 2013, p. 175).

\section{1 Agricultura Familiar na América Central}

Apesar das diferenças na definição dos estabelecimentos agropecuários familiares existentes em cada um dos países que compõem a região, o estudo FAO/CEPAL/IICA (2013) considerou agricultura familiar aquela unidade produtiva (e domicílio/hogar rural) que é dirigida por um agricultor por conta própria e que não contrata trabalhadores assalariados de maneira permanente. Essa definição também inclui as empresas agrícolas com menos de cinco pessoas ocupadas, aí incluídos o empregador e seus familiares não remunerados. Essa definição tornou possível a utilização de dados de diferentes fontes de pesquisas, tais como as encuestas (pesquisa amostrais) de hogares (domicílios), censos populacionais e censos agropecuários de diferentes países da região a fim de dimensionar as características da agricultura familiar (FAO/CEPAL/IICA, 2013, p. 182).

A região da América Central e México conta com mais 7,4 milhões de estabelecimentos agropecuários, sendo que, deste total, 5,8 milhões $(78,60 \%)$ podem ser considerados de agricultura familiar. Conforme a tabela 3 , verifica-se que o México é o país que possui maior número de estabelecimentos familiares (4.104.505), seguido pela Guatemala (830.684) e por El Salvador (397.433). É importante destacar que em todos os países da região, a proporção de estabelecimentos familiares em relação ao total de estabelecimentos agropecuários só é menor que $80 \%$ no México, país que possui maior extensão territorial e maior número de estabelecimentos. Nos demais países, a agricultura familiar representa mais de $80 \%$ do total de estabelecimentos, com destaque para Honduras, país no qual quase a totalidade dos estabelecimentos é de origem familiar $(97,20 \%)$. 
Tabela 3. Proporção de unidades agropecuárias familiares sobre o total de estabelecimentos agropecuários na América Central e México, segundo países

\begin{tabular}{cccc}
\hline Países & $\begin{array}{c}\mathbf{N}^{\circ} \text { de Total } \\
\text { Estabelecimentos }\end{array}$ & $\mathbf{N}^{\circ}$ de Unidades Familiares & $\begin{array}{c}\text { \% Unidades Familiares } \\
\text { sobre o total de } \\
\text { Estab. }\end{array}$ \\
\hline El Salvador & 397.433 & 325.895 & $82,00 \%$ \\
Guatemala & 830.684 & 718.585 & $86,50 \%$ \\
Honduras & 317.199 & 308.347 & $97,20 \%$ \\
México & 5.424 .428 & 4.104 .505 & $75,70 \%$ \\
Nicarágua & 268.527 & 223.374 & $83,20 \%$ \\
Panamá & 248.560 & 202.499 & $81,50 \%$ \\
\hline Total & 7.486 .831 & 5.883 .205 & $78,60 \%$ \\
\hline
\end{tabular}

Fonte: elaboração própria do autor a partir de Leporati et. al (2014).

De acordo com estudo da FAO/CEPAL/IICA (2013, p. 182), a agricultura familiar na América Central, excluindo-se o México, abriga mais de 2,4 milhões de famílias de agricultores, sendo que o país que possui maior quantidade de famílias é a Guatemala (pouco mais de um milhão), e o com menor quantidade a Costa Rica $(73 \mathrm{mil})$. Os dados mostram que o tamanho médio dos estabelecimentos familiares nessa região é de apenas 3,13 ha, sendo que no México esta média sobe para 6,83 ha enquanto que, no Panamá, cai para 1,35 ha.

Em grande parte dos países da região, a maioria dos agricultores trabalha de forma autônoma (por conta própria), utilizando apenas a própria força de trabalho e da família sem contratação de mão de obra assalariada. Contudo, nos últimos anos, vem ocorrendo um aumento dos trabalhadores assalariados que tem a agricultura como atividade secundária, assim como aqueles estabelecimentos que contratam um pequeno número de empregados.

Tabela 4. Superfície e tamanho médio dos estabelecimentos do setor agropecuário e na agricultura familiar na América Central e México

\begin{tabular}{ccccc}
\hline \multirow{2}{*}{ Países } & \multicolumn{2}{c}{ Setor Agropecuário (ha) } & \multicolumn{2}{c}{ Agricultura Familiar (ha) } \\
\cline { 2 - 5 } & Superfície & Média por Estab. & Superfície & Média por Estab. \\
\hline Guatemala & 5.315 .838 & 6,40 & 864.165 & 1,20 \\
México & - & 24,30 & - & 6,83 \\
Panamá & 2.698 .841 & 10,86 & 272.935 & 1,35
\end{tabular}


Total

8.014 .679

13,85

1.137 .100

3,13

Fonte: elaboração própria do autor a partir de Leporati et. al (2014).

De acordo com um outro estudo da FAO (2012a), em El Salvador e Guatemala, o percentual de trabalhadores que tem na agricultura uma atividade secundária é de $45 \%$, constituindo-se como o segundo maior grupo de agricultores familiares nestes países. Já na Costa Rica, o número de estabelecimentos que contratam até cinco trabalhadores é o segundo em importância, representando mais de uma quarta parte da agricultura familiar. Por sua vez, na região como um todo, a média de pequenos empregadores agrícolas em relação ao total de estabelecimentos com agricultura familiar é de 5\% (FAO/CEPAL/IICA, 2013, p. 183).

Tabela 5. Número de produtores agrícolas familiares na América Central, segundo categorias (por milhares pessoas)

\begin{tabular}{lcccc}
\hline \multicolumn{1}{c}{ Países } & $\begin{array}{c}\text { Conta } \\
\text { Própria }\end{array}$ & Peq. Empregadores & Outros & Total \\
\hline Guatemala & 564 & 20 & 478 & 1.062 \\
Honduras & 366 & 11 & 107 & 484 \\
Nicarágua & 226 & 17 & 91 & 334 \\
El Salvador & 115 & 19 & 96 & 230 \\
Panamá & 109 & 11 & 44 & 164 \\
Costa Rica & 55 & 21 & 3 & 79 \\
\hline
\end{tabular}

Fonte: elaboração própria do autor a partir de FAO/CEPAL/IICA (2013).

De forma geral, do total de agricultores familiares da América Central, $61 \%$ encontram-se na categoria de agricultores conta própria, para os quais a agricultura é a principal atividade, $4 \%$ correspondem a pequenos agricultores que contratam até cinco pessoas (contando os familiares não remunerados) e, finalmente, os $35 \%$ restantes correspondem a assalariados agrícolas e não agrícolas ou trabalhadores por conta própria não agrícolas que têm na agricultura uma atividade secundária.

\subsection{A agricultura familiar no Caribe}

Dentre todas as regiões que compõem a América Latina a subregião do Caribe é aquela que possui informações mais escassas em relação à agricultura familiar. Isto ocorre em face do pequeno número 
de países nos quais é possível encontrar dados sobre as características da agricultura, assim como, pelo fato de que em muitos deles o segmento da agricultura familiar não é diferenciado de outros setores.

Há ainda uma diversidade de definições existentes para a exploração agrícola na sub-região, sendo que, entre os países, observase uma grande variabilidade na definição de requisitos mínimos para definir o que seja uma exploração agrícola, o que dificulta a padronização dos dados e a análise das informações (GARNER Y DEL O CAMPOS, 2014 FAO/CEPAL/IICA, 2013, p. 192). Apesar dessas limitações, que impedem dimensionar a real importância do setor no desenvolvimento dos países, alguns esforços vêm sendo realizados no intuito de caracterizar os agricultores familiares da região e quantificáIos (SALCEDO Y GUZMÁN, 2014; GRAHAM, 2012).

A tabela 6 , a seguir, indica que a região do Caribe conta com um total de 1.507.757 unidades agropecuárias familiares, as quais representam $88,4 \%$ do total de unidades agropecuárias da região. Esse percentual é extremamente elevado, constituindo-se como o mais representativo entre todas as sub-regiões que compõem o território da ALC.

Tabela 6. Proporção de unidades agropecuárias familiares sobre o total de estabelecimentos agropecuários no Caribe, segundo países

\begin{tabular}{lccc}
\hline \multicolumn{1}{c}{ Países } & $\mathbf{N}^{\circ}$ de Total Estabelecimentos & $\begin{array}{c}\mathbf{N}^{\circ} \text { de Unidades } \\
\text { Familiares }\end{array}$ & $\begin{array}{c}\text { \% Unidades } \\
\text { Familiares sobre } \\
\text { o total de Estab. }\end{array}$ \\
\hline Antígua e Barbuda & 1.226 & 1.118 & 91,2 \\
Belize & 13.882 & 10.272 & 74,0 \\
Granada & 55.029 & 49.246 & 89,5 \\
Haiti & 1.018 .951 & 956.892 & 93,9 \\
Jamaica & 228.683 & 179.999 & 78,7 \\
Rep. Dominicana & 347.563 & 281.526 & 81,0 \\
Santa Lúcia & 9.972 & 8.287 & 83,1 \\
Suriname & 10.234 & 10.189 & 99,6 \\
Trinidad e Tobago & 19.111 & 10.228 & 53,5 \\
\hline Total & 1.704 .651 & 1.507 .757 & 88,4 \\
\hline
\end{tabular}

Fonte: elaboração própria do autor a partir de Leporati et. al (2014).

Na região do Caribe, destacam-se o Haiti, com quase um milhão de unidades familiares, as quais representam 93,9\% dos 
estabelecimentos do país, e a República Dominicana, que conta com 281.526 unidades agropecuárias familiares $(81,0 \%$ do total). Ainda que com estabelecimentos pouco numerosos, em países como o Suriname, o percentual de unidades agropecuárias familiares em relação ao total de estabelecimentos chega a quase $100 \%(99,6 \%)$.

\subsection{A Agricultura Familiar na América do Sul}

A agricultura familiar da América do Sul se caracteriza por uma grande heterogeneidade entre os estabelecimentos de agricultura familiar que possui. Ao visualizar-se a tabela 7 , verifica-se que o setor da agricultura familiar nos países corresponde entre $46,9 \%$ a $93,1 \%$ do total de estabelecimentos da região. Na média geral da região, os estabelecimentos com agricultura familiar correspondem a $78 \%$ do total de estabelecimentos agropecuários.

Ainda analisando esses dados, pode-se verificar que o Paraguai é - país no qual a agricultura familiar possui maior número de estabelecimentos em relação ao total, representando $93,1 \%$ de todos os estabelecimentos do país, seguido do Chile $(92 \%)$, Equador $(84,5 \%)$ e do Brasil (84,4\%). Somente no Uruguai, o grupo da agricultura familiar corresponde a menos da metade do total de estabelecimentos, correspondendo a $46,9 \%$.

Tabela 7. Proporção de unidades agropecuárias familiares sobre o total de estabelecimentos agropecuários na América do Sul, segundo países

\begin{tabular}{cccc}
\hline Países & $\begin{array}{c}\mathbf{N}^{\circ} \text { de Total } \\
\text { Estabelecimentos }\end{array}$ & $\begin{array}{c}\mathbf{N}^{\circ} \text { de Unidades } \\
\text { Familiares }\end{array}$ & $\begin{array}{c}\text { \% Unidades } \\
\text { Familiares sobre o } \\
\text { total de Estab. }\end{array}$ \\
\hline Colômbia & 2.021 .895 & 1.584 .892 & 78,4 \\
Equador & 842.882 & 712.035 & 84,5 \\
Peru & 2.213 .506 & 1.754 .415 & 79,3 \\
Argentina & 333.477 & 218.868 & 65,6 \\
Brasil & 5.175 .489 & 4.367 .902 & 84,4 \\
Chile & 301.269 & 277.166 & 92,0 \\
Paraguai & 289.649 & 269.559 & 93,1 \\
Uruguai & 44.890 & 21.038 & 46,9 \\
\hline Total & 11.223 .057 & 9.205 .875 & 78,0 \\
\hline
\end{tabular}

Fonte: elaboração própria do autor a partir de Leporati et. al (2014). 
Em relação à área ocupada pelo segmento da agricultura familiar na região, percebe-se, na tabela 8 , que os estabelecimentos agropecuários familiares sul-americanos possuem em média 28,9 ha. A Argentina é o país em que os estabelecimentos familiares possuem maiores áreas (107,45 ha), seguido do Chile (46,00 ha) e do Brasil $(24,17$ ha). Na direção oposta dos países de menor área média dos estabelecimentos familiares está o Peru (1,29 ha), o Equador (3,48 ha) e a Colômbia (4,48 ha).

Com relação à área total ocupada por esse segmento na região, os países podem ser divididos em pelo menos dois grupos: um primeiro grupo no qual a agricultura familiar corresponde a menos de uma quarta parte (25\%) da superfície agrícola (casos de Argentina, Uruguai, Paraguai e Brasil), e um segundo grupo no qual a agricultura familiar corresponde à metade ou mais da superfície agrícola (Equador, Chile e Colômbia). 
Tabela 8. Superfície e tamanho médio dos estabelecimentos do setor agropecuário e na agricultura familiar da América do Sul

\begin{tabular}{lcccc}
\hline \multirow{2}{*}{ Países } & \multicolumn{2}{c}{ Setor Agropecuário (ha) } & \multicolumn{2}{c}{ Agricultura Familiar (ha) } \\
\cline { 2 - 5 } & Superfície & Média por Estab. & Superfície & Média por Estab. \\
\hline Colômbia & 50.705 .453 & 25,08 & 7.105 .601 & 4,48 \\
Equador & 12.355 .831 & 14,70 & 2.481 .019 & 3,48 \\
Peru & 38.742 .465 & 17,50 & 2.268 .752 & 1,29 \\
Argentina & 174.807 .257 & 524,20 & 23.516 .942 & 107,45 \\
Brasil & 329.941 .393 & 63,75 & 105.581 .246 & 24,17 \\
Chile & 22.923 .754 & 85,00 & 11.703 .562 & 46,00 \\
Paraguai & 31.086 .894 & 107,33 & 2.816 .612 & 10,45 \\
\hline Total & 660.563 .047 & 119,65 & 155.473 .734 & 28,19 \\
\hline
\end{tabular}

Fonte: elaboração própria do autor a partir de Leporati et. al (2014).

Embora os dados ainda careçam de precisão e, sobretudo, de acompanhamento sistemático para análises longitudinais, é possível perceber com clareza que há uma presença numericamente significativa da agricultura familiar no universo social das unidades agropecuárias da ALC. Reunir e sistematizar esses dados é o primeiro passo para se reconhecer o seu potencial para o desenvolvimento rural.

\section{Oportunidades para a agricultura familiar na América Latina e no Caribe}

Os dados sobre a presença da agricultura familiar mostram que essa forma social de trabalho e produção é parte integrante da paisagem rural da ALC e integra as bases étnicas e culturais da diversidade social da sua população. A agricultura familiar tem desempenhado um papel crucial ao longo da história na região, uma vez que os arranjos familiares foram decisivos na formação da estrutura agrária.

Considerando a presença maciça da agricultura familiar nas sociedades da ALC, pode-se afirmar que o desenvolvimento econômico e social dessas sociedades dependerá do papel estratégico que for atribuído a este setor em cada país. Vale registrar e sublinhar que, em alguns países, especialmente na América Central, a agricultura familiar representa mais de $90 \%$ dos estabelecimentos rurais agrícolas. Portanto, políticas de apoio e de suporte são cruciais e estratégicas.

Em face de sua presença massiva e de seu potencial estratégico, a agricultura familiar poderá ter distintas contribuições para o 
desenvolvimento rural na ALC. No passado, a questão do excedente demográfico do setor rural era vista como um problema a ser superado porque criava obstáculos à industrialização (LIPTON, 2006). Mas, após o período dos ajustes estruturais da década de 1990, uma nova perspectiva sobre as funções da agricultura no desenvolvimento econômico emergiu e, atualmente, está claro que cabe à agricultura familiar um papel importante na manutenção da população nos espaços rurais. Mas a nova equação demográfica não é homogênea e possui dinâmicas distintas segundo países e regiões. Em algumas regiões rurais, a agricultura familiar continuará a fornecer força de trabalho para o setor de serviços e mesmo para migração, seja temporária ou permanente. Em outras, a permanência de parte da família e a manutenção da propriedade da terra mostra-se um importante dispositivo contra a crise que afetou os emigrantes desde 2008 nos países do Norte. A migração não é uma solução ou um caminho a ser preconizado para todos, assim como o velho discurso de "fixar o homem no campo" também perdeu sentido.

Um segundo papel que a agricultura familiar desempenha no desenvolvimento econômico refere-se à diversificação das economias locais. Esse processo pode ocorrer tanto por meio da pluriatividade como da agregação de valor aos produtos agrícolas. Há que se considerar que a existência de excedentes de mão de obra nas unidades familiares que não são inteiramente absorvidos pelas atividades agrícolas pode estimular o desenvolvimento de outras atividades e ocupações dentro e fora da propriedade. Essa é a explicação para o fato de que muitas das famílias de agricultores se tornaram pluriativos e possuem múltiplas fontes de ingresso (JANVRY e SADOULET, 2000; REARDON et al., 2001; SCHNEIDER, 2009).

O terceiro papel que a agricultura familiar pode exercer refere-se à preservação do patrimômio sociocultural, relacionado tanto à cultura imaterial como à língua, à gastronomia, e às manifestações folclóricas. Trata-se, verdadeiramente, da principal herança das sociedades camponesas que a agricultura familiar mantém, pois o modo de vida, a cultura e os valores camponeses conferem identidade social a muitas comunidades e povoados rurais. No contexto atual, muitas dessas heranças e valores são reorientados e inseridos em dinâmicas mercantis que acabam criando oportunidades econômicas relacionadas às atividades de turismo rural, gastronomia regional, festas e eventos 
rurais. Não são poucos os pequenos municípios ou comunidades rurais de camponeses e agricultores familiares que puderam redefinir seu portfólio de atividades e estratégias de ocupação da força de trabalho, atraindo turistas e pequenos empreendimentos. Os estudos mais interessantes sobre as dinâmicas territoriais e locais inovadores de desenvolvimento rural que tem sido feitos na América Latina nos últimos anos mostram abundantes evidências desse processo (BERDEGUÉ e BENITO, 2012; BERDEGUÉ et ali, 2012).

O quarto papel da agricultura familiar está relacionado à segurança alimentar. $\mathrm{O}$ aspecto mais importante e prioritário refere-se à contribuição da agricultura familiar à produção para autoconsumo e, secundariamente, ao abastecimento dos mercados locais, como feiras e mesmo ao poder público, mediante o fornecimento de alimentos para as escolas e compradores locais (D'ORICO, et.ali., 2014). Nesse sentido, é preciso enfatizar que malgrado não hajam restrições ou limites para que os agricultores familiares produzam para o mercado externo (como aliás, ocorre com o café nos países andinos, a soja, frangos e suínos no sul do Brasil, ou algumas frutas e verduras no México, etc), os mercados domésticos e locais serão sempre a primeira e mais importante opção para os produtos da agricultura familiar 6 . Não são apenas as distâncias menores e as quantidades pequenas de produtos que tornam os agricultores familiares mais competitivos nestes mercados locais.

No entanto, um quinto papel que a agricultura familiar desempenha refere-se à sua contribuição à segurança alimentar, à melhoria das condições de vida e à redução da pobreza na ALC, especialmente em áreas rurais (SCHUTER, 2014; IFAD / PNUD, 2013). O fortalecimento da agricultura familiar pode representar, em primeiro lugar, um aumento no excedente de alimentos disponíveis, quer pela melhoria da produção para consumo próprio nas fazendas ou pelo aumento da circulação local ou regional do excedente. Além disso, a melhoria das condições de alimentação pode impulsionar outras dimensões das condições de vida, como na saúde, educação e do próprio ambiente. É desnecessário enfatizar os efeitos positivos da

\footnotetext{
${ }^{6}$ As potencialidades e o papel dos mercados locais, assim como sua relação com o sistema agroalimentar dominante, é um tema de grande relevância que segue em aberto. Estudos de Reardon e Berdegué (2002) mostraram os limites dos mercados locais em fazer frente ao crescimento dos grandes supermercados em todas as regiões.
} 
melhoria da segurança alimentar e da nutrição na saúde e na educação. No entanto, é importante notar que uma população rural menos vulnerável e menos pobre também pode fazer uso mais sustentável dos recursos naturais como a água, o solo e a biodiversidade.

A sexta contribuição da agricultura familiar ao desenvolvimento rural poderá se dar no âmbito das estratégias de produção mais sustentáveis, como a redução significativa de insumos agroquímicos externos e formas de manejo vegetal e animal. Não há uma essência preservacionista per se na agricultura familiar vis-à-vis as formas não familiares. Mas pelo fato das unidades familiares em geral possuírem abundância do fator trabalho e não organizar a atividade econômica, seguindo uma racionalidade guiada pelo lucro, pode ser um fator favorável à transição ou mudanças no processo técnico produtivo. Nesse sentido, a agricultura familiar pode se tornar uma forma de produzir alimentos mais saudáveis e, com isto, angariar maior simpatia dos consumidores, o que pode inclusive abrir novos mercados e ajudar a solucionar alguns dos problemas com que se defronta (FAO, 2014a).

Por fim, há também razões de ordem social e cultural que não podem ser menosprezadas e nem esquecidas, pois as relações sociais de interconhecimento entre os produtores e compradores são importante característica dos mercados locais, que ajuda a reduzir a insegurança e os custos de transação, que se reflete em custos e preços finais relativos mais baixos. Do ponto de vista cultural, a produção de alimentos segundo as tradições e a dieta alimentar do local é um importante elemento de identidade e de coesão social de comunidades rurais, que não raro também passa a ser um atrativo não tangível do próprio meio rural, por meio do turismo rural. Nesse sentido, a agricultura familiar possui papel importante no fortalecimento das relações entre produtores e consumidores locais de alimentos que pode fortalecer não apenas o acesso aos alimentos, mas ativar uma série de outros elementos que acabam por ser centrais na reprodução das unidades familiares no meio rural.

\section{REFERÊNCIAS}

BARRIL, G.A.; ALMADA, F. 2007. La agricultura familiar en los países del Cono Sur. Asunción, Paraguay, IICA. 
BAUMEISTER, E. Construcción social del concepto de agricultura familiar con una visión Centroamericana. Working Paper, 2012.

BELIERES, J.F.; BONNAL, P.; BOSC, P.M.; LOSCH, B.; MARZIN, J.; SOURISSEAU, J.M. 2013. Les agricultures familiales du monde: définitions, contributions et politiques publiques. Montpellier, Francia, CIRAD.

BENGOA, J. (2003) 25 años de estudios rurales. Sociologias, Porto Alegre, a.5, n.10, jul/dez., p.36-99.

BERDEGUE, J.A.; BEBBINGTON, A.; ESCOBAL, J.; FAVARETO, A.; FERNANDEZ, M.I.; OSPINA, P.; RAVNBORG, H.M.; AGUIRRE, F.; CHIRIBOGA, M.; GOMEZ, I.; GOMEZ, L.; MODREGO, K.F.; PAULSON, S.; RAMIREZ, E.; SCHEJTMAN, A.; TRIVELLI, C. 2012. Territorios en Movimiento. Dinámicas Territoriales Rurales en América Latina. Documento de trabajo n. 110 , RIMISP, Santiago, Chile.

BERDEGUE, J.A.; BENITO, F.M. De Yucatán a Chiloé: dinâmicas territoriales en América Latina. Buenos Aires, Edición Teseo/RIMISP, $1^{\text {a }}$ ed., 2012.

CEPAL/FAO/IICA. Situación y perspectivas de la agricultura familiar en América Latina y el Caribe. In: Perspectivas de la agricultura y del desarrollo rural en las Américas: una Mirada hacia America Latina y el Caribe. San Jose/Costa Rica, IICA, 2013.

CIRAD. Les Agricultores familiales du monde - définitions, contributions et politiques publiques. Montepellier, CIRAD, 2013.

CONWAY, G. On Being A Smallholder. In: HAZELL,P. RAHMAN, A. New Directions for Smallhoder Agriculture. IFAD, Oxfrod University Press, Oxford, 2014, p.15-35

D'ODORICO P., CARR, J. , LAIO, F., RIDOLFI, L. \& VANDONI, S. 2014. Feeding humanity through global food trade, Earth's Future, 2, 458469. 
DE JANVRY, A. Y SADOULET, E. Income strategies among rural households in Mexico: the role of farm activities. World Development. 29: 467-480, 2001

DE JANVRY, A.; SADOULET, E. Rural poverty in Latin America. Determinants and exit paths. Food Policy 25: 389-409, 2000.

ECHENIQUE, J.; ROMERO, L. (eds.). Evolución de la agricultura familiar en Chile. Santiago, Chile, FAO, 2009

FAO (2007) (Organización de las Naciones Unidas para la Alimentación y la Agricultura); BID (Banco Interamericano de Desarrollo). Políticas para la agricultura familiar en América Latina y el Caribe. F. Soto Baquero; M.R. Fazzone; C. Falconi (eds.). Santiago, Chile, Oficina Regional de la FAO para América Latina y el Caribe, 2007

FAO (2011), "Agricultura familiar: evolución conceptual, desafíos y institucionalidad en América Latina y el Caribe", II Foro del Frente Parlamentario Contra el Hambre, 7 y 8 de junio, Bogotá (Colombia).

FAO (2012) Marco estratégico de mediano plazo de la FAO em: agricultura familiar en America Latina y El Caribe: 2012 - 2015. Documento de consulta con los países miembros. Vol. 14.0, Marzo 1, $2012 b, 45 p$.

FAO (2014a). The State of Food and Agriculture - Innovation in family farming. Rome, Italy, 161 pages

FAO (2014b) Agricultura Familiar em América Latina y El Caribe: recomendaciones de política. Santiago, Chile, 2014.

FAO, FIDA y PMA. (2013) El estado de la inseguridad alimentaria en el mundo 2013. Las múltiples dimensiones de la seguridad alimentaria. Roma, FAO.

FAO. 2015-2016 - The State of Agricultural Commodity Markets. FAO, Rome/ltaly, 2015 
FAO. 2013b. International year of family farming 2014. Master plan. Rome Disponível em: <http:// www.fao.org/fileadmin/user_upload/iyff/docs/Final Master Plan IYFF > Acesso em: 2014.

FIDA (Fondo Internacional de Politica Agrícola). La agricultura familiar en América Latina - Un nuevo análisis comparative. FIDA/RIMISP, Roma, 2014.

FRIEDMANN, H. Simple Commodity Production and Wage Labour in the American Plains. Journal of Peasant Studies, London, v.6, n.1, p.71-100, 1978.

GARNER, E. \& DE LA O CAMPOS, A. 2014. Identifying the "family farm": an informal discussion of the concepts and definitions. ESA Working Paper No. 14-10. Rome, FAO

GLADEK, E.; FRASER,M; ROEMERS, G; MUNOZ, O.S.; KENNEDY, E.; HIRSCH, P. The Global Food System - an analysis. WWF The Netherlands, 2016

GODFRAY, H. C. J., BEDDINGTON, J. R., CRUTE, I. R., HADDAD, L., LAWRENCE, D.,MUIR, J. F., et al. (2010). Food security. the challenge of feeding 9 billion people. Science, 327(5967), 812-818.

GRAEUB, B. E. et al. The State of Family Farms in the World, World Development (2015). Disponível em: < http://dx.doi.org/10.1016/j.worlddev.2015.05.012>

GRAHAM, B. Profile of the Small-Scale Farming in the Caribbean. Workshop on Small - Scale Farming in the Caribbean. FAO - Initiative hunger-free Latin American and the Caribbean. 2012. .

HAYAMI, Y.Family Farms and plantations in tropical development. Asian Development Bank, Vol.1 9 №.2, p. 70-89, 2002.

HAZELL,P; POULTON, C.; WIGGINS, S.; DORWARD, A. The Future of Small Farms: Trajectories and Policy Priorities. World Development, Vol.38, ${ }^{\circ}$ 
10, p.1349-1361, 2010.

HAZELL,P. RAHMAN, A. New Directions for Smallhoder Agriculture. IFAD, Oxfrod University Press, Oxford, 2014.

HLPE. 2013. Investing in smallholder agriculture for food security. A report by the High Level Panel of Experts on Food Security and Nutrition of the Committee on World Food Security, Rome.

IAASTD (International Assessment of Agricultural Knowledge, Science and Technology for Development). Agriculture at a crossroads - global report. Ed. Beverly D. McIntyre [et al.]. Washintong, 2009.

IFAD/UNEP. Smallholders, food security, and the environment. Rome/Italy, IFAD, 2013.

LEPORATI, M. et al. La agricultura familiar en cifras. In: SALCEDO, S; y GUZMÁN, L. (Ed.) Agricultura Familiar em América Latina y El Caribe: recomendaciones de política. Parte 01, Cap. 2, p. 35-56, 2014.

LIPTON, M. The family farm in a globalizing world: The role of crop science in alleviating poverty. 2020 Discussion Paper No. 40. Washington, D.C.: International Food Policy Research Institute, 2005.

LIPTON, M. Can small farmers survive, prosper, or be the key channel to cut mass poverty? The Electronic Journal of Agricultural and Development Economics 3(1), 58-85, 2006.

LOWDER, S. K. et al. The Number, Size, and Distribution of Farms, Smallholder Farms, and Family Farms Worldwide. World Development (2016).

Disponível em: <http://dx.doi.org/10.1016/j.worlddev.2015.10.041>

LOWDER, S.K., SKOET, J. and SINGH, S. What do we really know about the number and distribution of farms and family farms worldwide? Background paper for The State of Food and Agriculture 2014. ESA Working Paper No. 14-02. Rome, FAO, 2014. 
MANZANAL, M.; NEIMAN, G. (coords.). Las agriculturas familiares del Mercosur. Buenos Aires, Argentina, Ediciones Ciccus, 2010.

NAGAYETS, O. 'Small farms: Current status and key trends'. In: The Future of Small Farms. Proceedings of a Research Workshop, Wye, UK, June 26-29. Washington, DC: International Food Policy Research Institute, 2005.

PAULILO. M.I.S. FAO, Fome e Mulheres Rurais. DADOS - Revista de Ciências Sociais, Rio de Janeiro, vol. 56, n. 2, pp. 285 a 310, 2013.

PERACI, A.S. Agricultura familiar: Evolución conceptual, desafíos e institucionalidad. In V Reunión del Grupo de Trabajo (GT2025), Iniciativa América Latina y Caribe sin Hambre 2025. Lima, Perú, FAO, 2011.

PIADAL (Panel Independiente de Agricultura para el Desarrollo en América). 2013. Agricultura y desarrollo en América Latina: gobernanza y políticas públicas. Barrantes, R.; Berdegué, J; Janvry, A. de et al. Buenos Aires, Argentina, Teseo, 2013.

PLOEG, J. V der. Camponeses e impérios alimentares: lutas por autonomia e sustentabilidade na era da globalização. POA, Ed. UFRGS, 2008.

PLOEG, J. V der. Peasants and the art of farming - a chayanovian manifesto. Hallifax/Winnipeg/Canada, Fenwood Publishing, 2013

PONISIO LC, M'GONIGLE LK, MACE KC, PALOMINO J, DE VALPINE P, KREMEN C. Diversification practices reduce organic to conventional yield gap. Proc. R. Soc. B 282:20141396, 2015.

PRETTY, J. et alii. The top 100 questions of importance to the future of global agriculture. International Journal of Agricultural Sustainability 8(4), p. 219-236, 2010.

PRETTY, J., TOULMIN, C. \& WILLIAM, S. 2011 . Sustainable intensification in African agriculture. International Journal of Agricultural Sustainability, 9(1): 3-4. 
RAMOS, A (2014) Compilation of the definitions and methodologies commonly used and accepted at a national, regional and international level to define and describe family farming based on its diversity. Final Report of the International Working Group on Common Criteria for achieving definitions and typologies on Family Farming (IWG FF). November 29, 2014

REAF - REUNIÃO ESPECIALIZADA DA AGRICULTURA FAMILIAR DO MERCOSUL. Bases para o reconhecimento e identificação da agricultura familiar no Mercosul. MERCOSUR/ VI REAF/ DT N ${ }^{\circ}$ 03/06. ANEXO IX. 2006a. In: <http://www.mda.gov.br/reaf/>. Acesso em: 25 out. 2011.

REARDON, T., BERDEGUÉ, J.A., ESCOBAR, G. (Eds.).Rural Nonfarm Employment and Incomes in Latin America. Special Issue of World Development 29 (3): 395 - 573, 2001.

REARDON, T.; BERDEGUÉ,J.A. The rapid rise of supermarkets in Latin America. Challenges and opportunities for development'. Development Policy Review, 20 (4): 371-388.

REGANOLD, J.P.; WACHTER, J. M. Organic agriculture in the twenty-first century. Nature Plants. Vol 2, February 2016.

SABOURIN, E.; SAMPER, M.; SOTOMAYOR, O. Políticas públicas y agriculturas familiares en América Latina: balance, desafíos y perspectivas. Santiago de Chile, ed. CEPAL, Red PPAL, IICA, 2014

SALCEDO, S.; GUZMÁN, L. (eds.). Agricultura familiar en América Latina y el Caribe: Recomendaciones de política. Santiago, Chile, FAO, 2014.

SCHEJTMAN, A. Alcances sobre agricultura familiar en América Latina. Diálogo Rural Iberoamericano. Documento de trabajo n. ${ }^{\circ} 21$. San Salvador, El Salvador, Programa Dinámicas Territoriales Rurales, RIMISP, 2008

SCHEJTMAN, A. Alcances sobre agricultura familiar en América Latina. Diálogo Rural Iberoamericano. Documento de trabajo No. 21. San 
Salvador, El Salvador: Programa Dinámicas Territoriales Rurales, RIMISP, 2008

SCHNEIDER, S. A agricultura familiar na América Latina: relatório-síntese a partir de 6 países. Reporte sobre la agricultura familiar en América Latina. Análisis de pobreza y desigualdad rural en América Latina. Santiago, Chile, FIDA, RIMISP, 2013.

SCHNEIDER, S. Family farming in Latin America and the Caribbean. Deep Roots. 1ed.Roma: FAO, v. 1, p. 26-29, 2014.

SCHNEIDER, S., NIEDERLE, P. A. Resistance strategies and diversification of rural livelihoods: the construction of autonomy among Brazilian family farmers. Journal of Peasant Studies. , v.37, p.379- 405, 2010.

SCHNEIDER, S.; ESCHER, F. La construción del concepto de agricultura familiar en America Latina. FAO-Chile, unpublished paper, 2012.

SCHULTZ, T.W. Transforming Traditional Agriculture. New Haven, CT: Yale University Press, 1964.

SCHUTTER, O. The transformative potential of the right to food. Report of the Special Rapporteur on the right to food. Human Rights Council, 25th Session. New York: U.N. General Assembly, 2014.

SILVA, J.F.G.; GÓMEZ, J.S.; CASTAÑEDA, R. (eds.). Boom agrícola y persistencia de la pobreza rural. Santiago, Chile, Oficina Regional de la FAO para América Latina y el Caribe, 2009.

SOTO BAQUERO, F., M. RODRÍGUEZ FAZZONE, AND C. FALCONI . Políticas para la Agricultura Familiar en América Latina y el Caribe. Santiago, Chile: FAO-BID, 2007.

WARMAN, A. "Los estudios campesinos veinte años después." Revista del Banco de Comercio Exterior de México 38(8), 1988. 
WIGGINS, S. Can the Smallholder model deliver poverty reductionand food security for rapidly growing population in Africa? FAC, Working Paper Nº8, Overseas Development Department, London, 2009.

WIGGINS, S.; J. KIRSTEN, J; LLAMBÍ, L. 'The future of small farms'. World Development 38 (10): 1341-1348, 2010.

WOLF, E. Sociedades Camponesas. Rio de Janeiro, Brazil: Zahar Editores, 1976.

WOLF, E. Types of Latin American Peasantry: A Preliminary Discussion. American Antropologist, Vol. 57, n 3, p. 452-471, 1955.

Submetido em 30/05/2016

Aprovado em 27/06/2016

Sobre o Autor

Sergio Schneider

Professor Associado da Universidade Federal do Rio Grande do Sul, Departamento de Sociologia e membro permanente dos Programas de Pós-Graduação em Desenvolvimento Rural (PGDR) e de Sociologia - PPGS.

E-mail: schneide@ufrgs.br 